\title{
Left Atrial Systolic Function Assessed by Left Atrial Ejection Force in Patients with Sick Sinus Syndrome and Paroxysmal Atrial Fibrillation
}

\author{
Takashi TOKUSHIMA, MD, Toshinori UTSUNOMIYA, MD, \\ Kazuyo YoshIDA, MD, Keiko KIDO, MD, Toshihiro OGAwA, MD, \\ Toshihiro RYU, MD, Toru OgatA, MD, Shinsuke TsuJ, MD, \\ and Shuzo MATSUO, MD
}

\begin{abstract}
SUMMARY
We evaluated left atrial systolic function using left atrial ejection force (LAEF) in 19 patients with sick sinus syndrome (SSS) and in 20 with paroxysmal atrial fibrillation (Paf) whose ages ranged from 48 to 80 years. We also evaluated 35 normal individuals for comparison. The LAEF was calculated as $1 / 3 \times$ mitral valve area $\times$ (peak velocity of A wave) $)^{2}$ using two-dimensional and pulsed-Doppler echocardiography according to Newton's law of motion and hydrodynamics. In normal individuals, LAEF positively correlated with age $(r=0.82, p<0.01)$. Normal LAEF was calculated as $0.098 \times$ age -0.74 (kdynes) from the regression line. Because of this correlation, we used age-corrected LAEF $(\% \mathrm{LAEF})$ that was calculated as (measured LAEF / normal LAEF) $\times 100$. The results showed that this value was $53 \pm 26 \%$ in patients with SSS and $54 \pm 26 \%$ in patients with Paf. Both were significantly lower than normal individuals $(p<0.001)$. Among SSS subtypes, \%LAEF was lower in types II and III than in type I $(51 \pm 14 \%, 37 \pm 19 \%$, and $81 \pm 35 \%$, respectively). In conclusion, left atrial systolic function is depressed in patients with Paf and SSS, in particular, types II and III. These results suggest that the pathological abnormalities extend not only to the sinus node but also to the left atrial muscle in patients with SSS and Paf. (Jpn Heart J 2000; 41: 723-732)
\end{abstract}

Key words: Sick sinus syndrome, Paroxysmal atrial fibrillation, Left atrial systolic function, Left atrial ejection force

SICK sinus syndrome (SSS) is defined as a disorder of sinus nodal function. Clinically, electrocardiograms of patients with SSS show sinus bradycardia, sinus arrest, sinoatrial (SA) block and sometimes episodes of tachycardia such as paroxysmal atrial fibrillation (Paf) and paroxysmal supraventricular tachycardia. Pathological studies have revealed histological abnormalities around the sinus

From the Division of Cardiology, Department of Internal Medicine, Saga Medical School, Saga, Japan.

Address for correspondence: Takashi Tokushima, MD, Department of Internal Medicine, Division of Cardiology, Saga Medical School, 5-1-1 Nabeshima, Saga 849-8501, Japan.

Received for publication June 21, 2000.

Revised and accepted August 25, 2000. 
node, and sometimes around the atrio-ventricular node or right atrium, ${ }^{1 \sim 4)}$ which is called binodal disease. ${ }^{5)}$

Nawata, et al. $\left.{ }^{6}\right)$ noted left atrial systolic dysfunction in patients with SSS, probably due to pathological abnormalities in the left atrial (LA) muscle. However, to clinically assess LA systolic function noninvasively is difficult. Recently, Manning, et al. ${ }^{7}$ described a new method with which to assess LA systolic function that consists of measuring left atrial ejection force (LAEF) using two-dimensional and pulsed-Doppler echocardiography. The LAEF is then calculated from Newton's second law of motion. The present study measures LAEF in normal individuals and in patients with SSS and Paf to assess systolic LA function.

\section{SubJECTS}

We examined 19 patients with SSS, 20 with Paf and 35 normal individuals. Sick sinus syndrome was diagnosed by 12-lead electrocardiography, 24-hour ambulatory monitoring and an electrophysiological study. According to Rubenstein, et al., ${ }^{8}$ type I was defined as persistent sinus bradycardia of less than $50 / \mathrm{min}$ that was not influenced by drugs or by vagal tonus, type II was defined as sinoatrial block or sinus arrest and type III was characterized by bradycardia-tachycardia.

Patients with mitral regurgitation (more than mild), aortic regurgitation (more than mild), left ventricular hypertrophy and left ventricular systolic dysfunction were excluded because these conditions can affect the velocity of atrial ejection flow. The ages of the patients with SSS and Paf and the normal individuals ranged from 48 to $80(67 \pm 10), 44$ to 79 $(66 \pm 9)$ and 14 to $77(42 \pm 17)$ years, respectively.

\section{MethodS}

Principle of left atrial ejection force (LAEF): According to Newton's law of motion, force equals mass times acceleration. Therefore, Manning, et al. ${ }^{7}$ surmised that LAEF is equal to left ejection volume times acceleration of left atrial flow. They used the formula; $\mathrm{LAEF}=1 / 2 \times \mathrm{MVA} \times \mathrm{A}^{2}$, where MVA is the mitral valve area and $A$ is the velocity of atrial filling. However, this formula cannot correctly calculate LAEF because whereas Newton's low of motion can be applied to a solid mass, it cannot be applied to a fluid. Hydrodynamically, force equals consistency of the fluid $(\sigma)$ multiplied by the area of discharge $(\rho)$ multiplied by flow velocity squared $(q)$. Because the velocity of an atrial filling (A) wave changes during 
transmitral flow, mean force is calculated as follows;

$$
\begin{aligned}
\text { mean force } & =\frac{1}{\Delta t} \int_{0}^{\Delta t} \rho q^{2} \sigma d t=\frac{\rho \sigma}{\Delta t} \int_{0}^{\Delta t}\left[(\mathrm{~A} / \Delta \mathrm{t}) \mathrm{t}^{2}\right] \mathrm{dt}=\frac{\rho \sigma \mathrm{A}^{2}}{\Delta \mathrm{t}^{3}} \int_{0}^{\Delta \mathrm{t}} \mathrm{t}^{2} \mathrm{dt} \\
& =\frac{\rho \sigma \mathrm{A}^{2}}{\Delta \mathrm{t}^{3}}\left[\frac{1}{3} \mathrm{t}^{3}\right]_{0}^{\Delta \mathrm{t}}=\frac{1}{3} \rho \sigma \mathrm{A}^{2}
\end{aligned}
$$

where $\sigma$ is $1.06 \mathrm{~g} / \mathrm{m} l$ and $\rho$ is MVA. Therefore, the final formula is as follows; $\mathrm{LAEF}=1 / 3 \times \mathrm{MVA} \times \mathrm{A}^{2}$. (Personal communication with Motoaki Sugawara, Professor, Department of Cardiovascular Sciences, Tokyo Women's Medical School College, Japan)

Echocardiography: Echocardiograms were recorded using an SSH-160A ultrasonic machine (Toshiba Co., Ltd., Tokyo, Japan) and a $2.5 \mathrm{MHz}$ transducer with the subject in the left decubitus position. Mitral valve diameter (MVD) was measured manually at the mid-portion of the A wave from a four-chamber view using two-dimensional echocardiography. Mitral valve area (MVA) was calculated as $3.14 \times(\mathrm{MVD} / 2)^{2}$ on the assumption that the mitral valve orifice is circular. Sample volume was placed at the mitral annulus level and A waves were recorded from a four-chamber view at a paper speed of $50 \mathrm{~mm} / \mathrm{sec}$ using pulsed-Doppler echocardiography. The peak velocity of A waves was obtained from averaging three consecutive beats.

Statistical analysis: Data are expressed as means \pm standard deviation. Student's $t$-test was used to analyze unpaired data. Linear regression analysis of scatter data was performed using the least squares method. Statistical significance was defined as a $p$-value below 0.05 .

\section{RESULTS}

Normal individual: Figure 1 shows a four-chamber echocardiogram obtained in the two-dimensional mode (left panel) and transmitral flow determined in the pulsed-Doppler mode (right panel) from a normal individual (65 year-old male). Manual tracing determined that the diameter of the mitral orifice was $25 \mathrm{~mm}$. The MVA value was calculated as 4.9 $\mathrm{cm}^{2}\left[=3.14 \times(25 / 2)^{2}\right]$. The peak velocity of the A wave was $57 \mathrm{~cm} / \mathrm{sec}$. Therefore, the calculated LAEF value for this individual was 5.3 kdynes.

Relationship between age and the LAEF in normal individuals: Figure 2 shows the relationship between age and LAEF in 35 normal individuals. Age and LAEF were significantly correlated $(r=0.82, p<0.01)$. From the regression line, we calculated normal LAEF as LAEF $=0.098 \times$ age (year) -0.74 (kdynes). We defined age-corrected LAEF (\%LAEF) as \%LAEF = 
4 chamber view ( $\mathrm{B}$ mode )

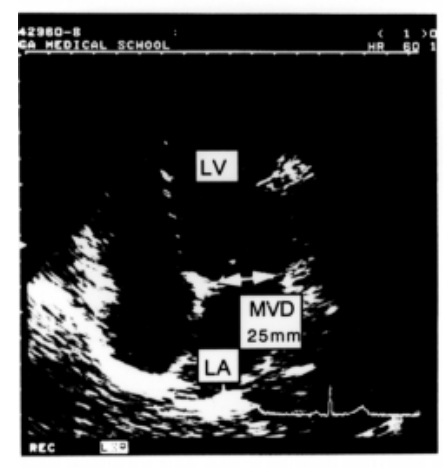

$$
\begin{aligned}
& \text { MVD }=25 \mathrm{~mm} \\
& \text { MVA }=4.9 \mathrm{~cm}^{2}
\end{aligned}
$$

$$
\text { Transmitral flow ( Pulsed wave Doppler, PWD) }
$$

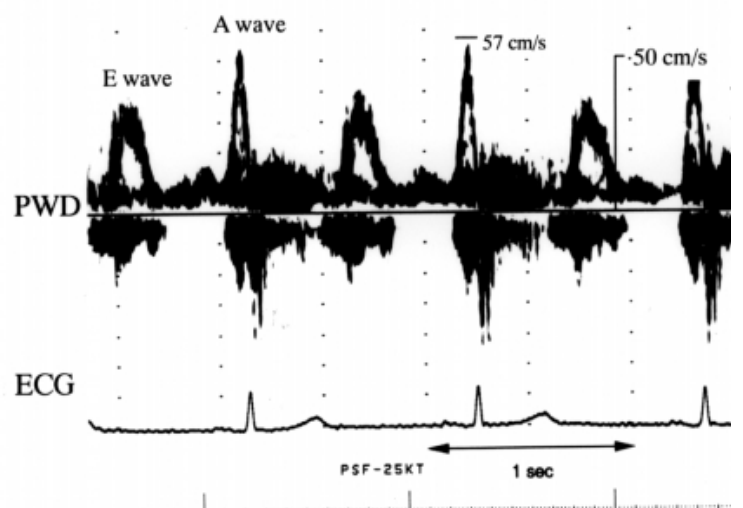

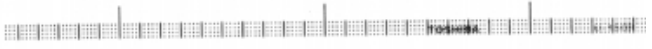

$$
\begin{aligned}
\text { LAEF } & =1 / 3 \times \text { MVA } \times A^{2} \\
& =1 / 3 \times 4.9 \times 57^{2} \\
& =5.3 \text { kdynes }
\end{aligned}
$$

Figure 1. Normal individual. Mitral valve diameter (MVD) of this 65 year-old normal male was measured by two-dimensional mode echocardiography with four-chamber view (left panel). Mitral valve area (MVA) was calculated as $4.9 \mathrm{~cm}^{2}$. Peak velocity of A wave was measured by pulsed-Doppler echocardiography (right panel). Calculated left atrial ejection force (LAEF) was 5.3 kdynes.

(measured LAEF / normal LAEF) $\times 100$.

Example of patient with SSS: Figure 3 shows a four chamber view echocardiogram obtained in the two-dimensional mode (left panel) and transmitral flow determined in the pulsed-Doppler mode (right panel) from a 68 yearold female with SSS type II. The LAEF value calculated using the above formula was 2.5 kdynes. Since the normal LAEF of a 68 -year-old was 5.9 kdynes calculated from the regression line, the \%LAEF of this patient was $42 \%$.

Value of LAEF in patients with SSS and Paf: Because the mean ages in patients with SSS and Paf (67 and 66 years, respectively) were higher than those of the normal volunteers (42 years), we compared \%LAEF among the three groups. Figure 4 shows that \%LAEF was significantly smaller in patients with SSS and Paf than in normal individuals (53 $\pm 26 \%$, $54 \pm 26 \%$ vs $103 \pm 43 \%, p<0.001$, respectively). However, \%LAEF did not differ between SSS and Paf.

LAEF in patients with SSS type I, II and III: Figure 5 shows \%LAEF in patients with types I, II and III SSS. The \%LAEF tended to be smaller in patients with types II and III than type I $(54 \pm 14 \%, 37 \pm 19 \%$, vs 81 


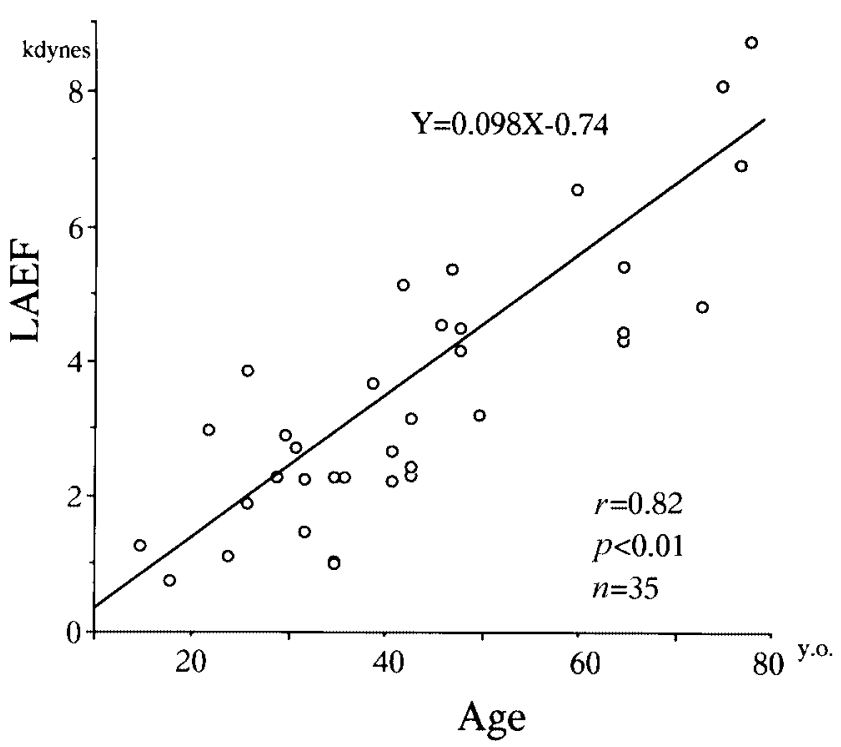

Figure 2. Relationship between LAEF and age in normal individuals.

LAEF positively correlated with age $(r=0.82, p<0.01)$ in normal individuals.

4 chamber view ( B mode )

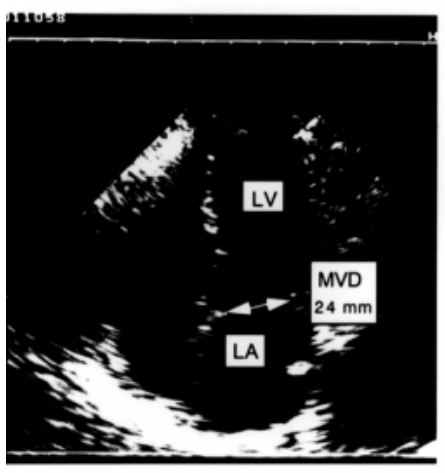

$$
\begin{aligned}
\text { MVD } & =24 \mathrm{~mm} \\
\text { MVA } & =4.5 \mathrm{~cm}^{2} \\
\text { LAEF } & =1 / 3 \times \text { MVA } \times A^{2} \\
& =1 / 3 \times 4.5 \times 41^{2} \\
& =2.5 \text { kdynes }
\end{aligned}
$$

Transmitral flow ( PWD )

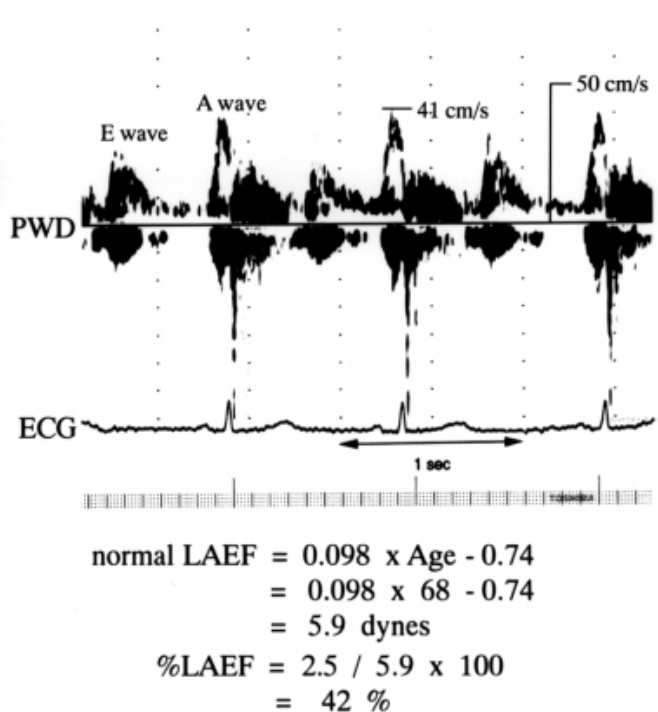

Figure 3. Patient with SSS. Calculated LAEF in this 68 year-old woman with SSS, type II was 2.5 kdynes and age-corrected LAEF (\%LAEF) was $42 \%$. LA systolic function was depressed compared with normal individuals. 


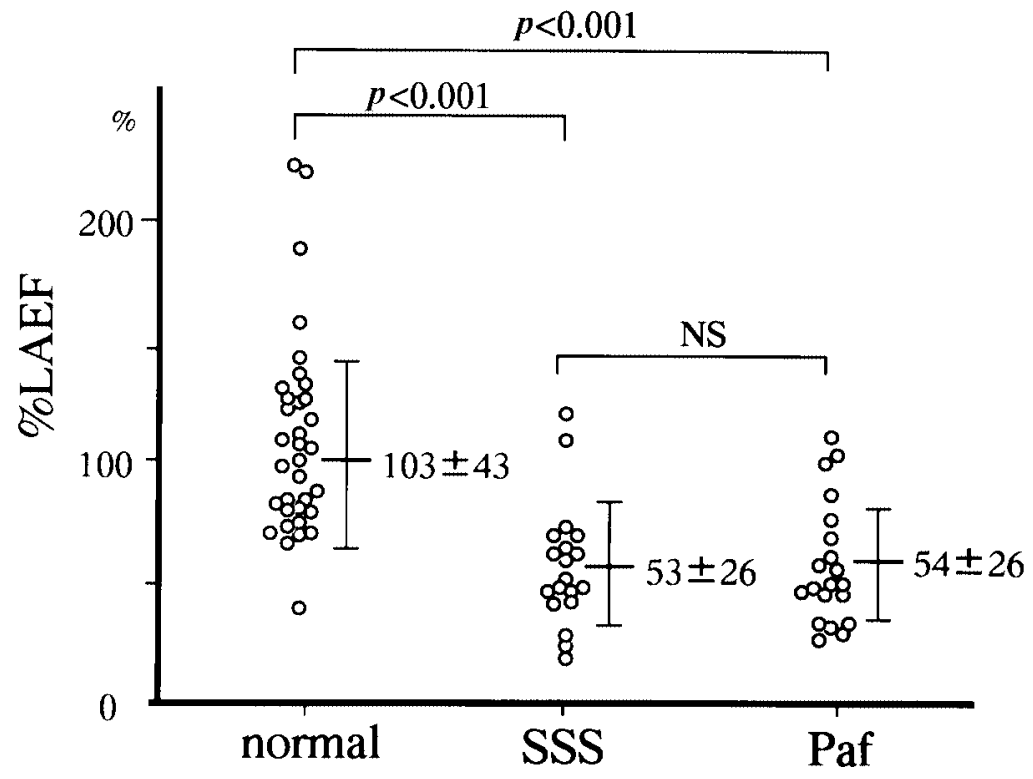

Figure 4. Comparison of \%LAEF among normal individuals and patients with SSS and Paf. Value of \%LAEF was depressed in patients with SSS and Paf compared with normal individuals $(53 \pm 26 \%, 54 \pm 26 \%$ vs $103 \pm 43 \%, p<0.001$, respectively).

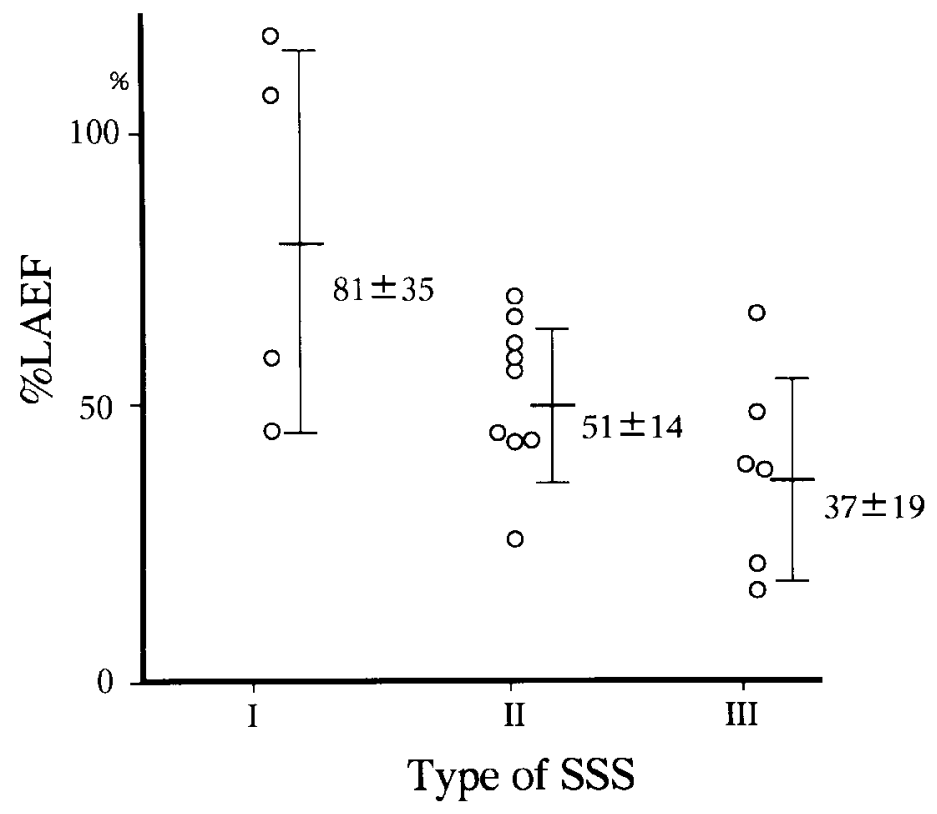

Figure 5. Comparison of \%LAEF in SSS subtypes. Value of \%LAEF was smaller in patients with type II and type III than with type I $(54 \pm 14 \%, 37 \pm 19 \%$, vs $81 \pm 35 \%$, respectively). 
$\pm 35 \%$, respectively). However, these values were not statistically significant.

\section{DISCUSSION}

Atrial systolic function in patients with SSS and Paf: Nawata, et al. ${ }^{6)}$ reported that left atrial active contraction was significantly impaired in several patients with SSS, especially when accompanied by bradycardia-tachycardia. Mattioli, et al. ${ }^{9)}$ reported that stroke occurred in 15 of 80 patients with SSS who had an implanted pacemaker, and they indicated that one reason for this could be a low atrial ejection force. Their report suggested that pathological abnormalities extend to the atrial muscle in some patients with SSS. Thery, et al. $\left.{ }^{3}\right)$ reported that lesions of the atrial muscle such as hemorrhage, fiber degeneration or fibrosis were associated with sinus nodal cell deficiency in 4 of 6 patients with bradycardia-tachycardia. Demoulon, et $a l .{ }^{4}$ ) also found extensive atrial fibrosis with loss of muscle and architecture in 5 of 6 patients with sinus arrest, sinoatrial block or bradycardia-tachycardia. The present study demonstrated using noninvasive left atrial ejection force (LAEF), that LA systolic function had deteriorated in patients with SSS as well as in those with Paf. Also, the degree of LA dysfunction tended to differ among SSS subtypes (Figure 5), indicating that pathological abnormalities in LA muscle were most prominent in patients with SSS type III.

Evaluation of atrial systolic function: A method with which to evaluate left atrial systolic function noninvasively and accurately has not been established because of complexity and hemodynamics. Some investigators have applied left atrial wall motion, left atrial volume or systolic time interval by M-mode echocardiography. ${ }^{11-14)}$ Flow velocity at the left atrial appendage recorded by pulsed-Doppler using transesophageal echocardiography has emerged as a useful method. ${ }^{15)}$ Manning, et al. ${ }^{7)}$ introduced LAEF, which is easily calculated from transthoracic echocardiography and can be compared among individuals over time. They also stated that this value is not affected by preload or afterload. They placed a sample volume at the mitral tip to measure the peak velocity of $\mathrm{A}$ waves and used the formula " $1 / 2 \times \mathrm{MVA} \times \mathrm{A}^{2}$ " to calculate LAEF. However, this method would not be accurate because transmitral flow cannot be assumed to behave as a solid mass. Other methods using left atrial ejection fraction and systolic time interval by M-mode echocardiography did not correlate with left atrial ejection force (LAEF) in our study. LAEF can be clinically used as an independent parameter of the LA systolic function index in heart 
diseases. ${ }^{16)}$

Aging and left atrial systolic function: According to the present results and those of Mattioli, et al., ${ }^{17)}$ age considerably influences LAEF. Increased atrial systolic function appears to be the compensatory process for agerelated left ventricular diastolic dysfunction. Therefore, the age-corrected LAEF (\%LAEF) is necessary when comparing LAEF among various ages. Since the relationship between LAEF and age is linear $(r=0.82, p<0.05)$ in normal individuals (Figure 2), we used age-corrected LAEF (\%LAEF) to evaluate LA systolic function.

Influence of blood flow on pulmonary veins: Left atrial contraction produces both the forward blood flow towards the LV through the mitral valve and backward flow towards the pulmonary veins (PV). Backward flow can be observed using transesophageal or transthoracic color or pulsed-Doppler echocardiography. This flow increases in patients with elevated left ventricular end-diastolic pressure (LVEDP). ${ }^{18-20)}$ When evaluating left atrial systolic function, flow towards the pulmonary veins (backward flow) cannot be ignored. We have examined "backward LAEF" in 45 patients with heart disease and in normal individuals. ${ }^{21)}$ In that study, backward LAEF was calculated by transthoracic echocardiography using the diameter of the pulmonary vein and the peak velocity of backward atrial flow. Backward LAEF (the sum of 4 PVs) was only $12.9 \pm 6.1 \%$ of the forward LAEF and total LAEF (forward plus backward ejection force) was closely correlated with forward LAEF $(r=0.99, p<0.001)$.

Study limitations: Evaluation of LA systolic function using only forward flow (transmitral flow) may be inaccurate when LVEDP elevates and backward flow increases. One report indicates that atrial systolic function is impaired just after electrical or chemical cardioversion in patients with Paf. ${ }^{10)}$ Since Paf is often seen in patients with SSS type III, LAEF may be influenced by the duration of Paf in addition to histological LA damage. However, this period is impossible to check because some patients are asymptomatic during atrial fibrillation. We assumed that the shape of the mitral annulus is circular to calculate MVA. The actual shape, however, is not circular and it changes with the cardiac cycle. Although the relationship between LAEF and age was linear $(r=0.82)$, the variance was relatively large at the median age of normal individuals. This may be due to other factors that were not clarified in our study.

In conclusion, LAEF was smaller in patients with SSS types II, III and Paf than in normal individuals. These results suggest that pathological abnormalities are present in the left atrial muscle in patients with SSS and Paf, and extend not only to the conduction system but also to the LA mus- 
cle in some patients with SSS. We conclude that LAEF can evaluate LA muscular involvement.

\section{REFERENCES}

1. Kaplan BM, Langendorf R, Lev M, Pick A. Tachycardia-bradycardia syndrome (so-called "sick sinus syndrome) pathology, mechanism and treatment. Am J Cardiol 1973; 31: 497-508.

2. Bharati S, Nordenberg A, Bauernfiend R, et al.. The anatomic substrate for the sick sinus syndrome in adolescence. Am J Cardiol 1980; 46: 163-72.

3. Thery C, Gosselin B, Lekieffre J, Warembourg H. Pathology of sinoatrial node correlation with electrocardiographic findings in 111 patients. Am Heart J 1977; 93: 735-40.

4. Demoulin JC, Kulbertus HE. Histological correlates of sinoatrial disease. Br Heart J 1978;40:1384-9.

5. Ferrer MI. The sick sinus syndrome. Circulation 1973;57:635-41.

6. Nawata T, Toma Y, Date T, et al. Study of atrial contraction in sick sinus syndrome using conventional and esophageal echocardiography. J Cardiogr 1983; 13: 981-90.

7. Manning WJ, Silverman DI, Katz SE, Douglas PS. Atrial ejection force: a noninvasive assessment of atrial systolic function. J Am Coll Cardiol 1993; 22: 221-5.

8. Rubenstein JJ, Schulman CL, Yurchak PM, DeSanctis RW. Clinical spectrum of the sick sinus syndrome. Circulation 1972; 56: 5-13.

9. Mattioli AV, Castellani ET, Paolillo C, Fusco A, Molinari R, Palladini G, et al. Stroke in pacemaker users for sinus node disease. Relevance of atrial function and clinical characteristics (Italian with English abstract). Cardiologia 1995; 40: 123-8

10. Mattioli AV, Tarabini CE, Vivoli D, Molinari R, Mattioli G. Restoration of atrial fibrillation of different etiological origins. Cardiology 1996; 25:205-11

11. Sasse L. Echocardiography of left atrial wall. JAMA 1974; 228: 1667.

12. Yoshikawa J, Kato H, Owaki T, Tanaka K. Study of posterior left atrial wall motion by echocardiography and its clinical application. Jpn Heart J 1975; 16: 683-693

13. Strunk BL, Fitzgerald JW, Lipton M, Popp RL, Barry WH. The posterior aortic wall echocardiogram: Its relationship to left atrial volume change. Circulation 1975; 54: 744-50.

14. Abe H, Yokouchi M, Deguchi F, Saitoh F, et al. Measurement of left systolic time intervals in hypertensive patients using Doppler echocardiography: Relation to fourth heart sound and left ventricular wall thickness. J Am Coll Cardiol 1988; 11: 800-5.

15. Pozzoli M, Febo O, Torbicki A, et al. Left atrial appendage dysfunction: A cause of thrombosis? Evidence by transesophageal echocardiography-Doppler studies. J Am Soc Echocardiogr 1991; 4: 435-41

16. Gotzsche O, Darwith A, Hansen LP, Gotzshe. Abnormal left ventricular diastolic function during cold pressor test in uncomplicated insulin-dependent diabetes mellitus. Clinical Science 1995; 89: 461-5.

17. Mattioli AV, Tarabini CE, Vivoli D, Molinari R, Mattioli G. Atrial ejection force: findings in healthy subjects (Italian with English abstract). Cardiologia 1995; 40: 341-5.

18. Takeuchi Y, Yokota Y, Tsumura M, et al. Left atrial ejection performance in heart failure assessed by transesophageal Doppler echocardiography (Japanese with English abstract). J Cardiology 1992; 22: 193-200.

19. Stojnic BB, Redjen GS, Perisic NJ, Pavlovic PB, Stosic JJ, Prcovic M. Pulmonary venous flow pattern studied by transesophageal pulsed Doppler echocardiography in mitral stenosis in sinus rhythm: effect of atrial systole. European Heart J 1993; 14: 1597-601.

20. Takeuchi Y, Yokota W, Yokoyama Y. Left atrial backward ejection in symptomatic hypertrophic cardiomyopathy. Assessment by transthoracic and transesophageal Doppler echocardiography. Jpn Circ J 1994; 58: 809-20.

21. Tokushima T, Utsunomiya T, Morooka T, et al. Assessment of left atrial muscular involvement using the left atrial ejection force in 142 patients with heart disease (Abstract). J Am Soc Echocardiogr. 1997; 10: 424. 